\title{
Vibration Control With MEMS Electrostatic Drives: A Self-Sensing Approach
}

\author{
Steven Ian Moore and S. O. Reza Moheimani, Fellow, IEEE
}

\begin{abstract}
Nanopositioning is the actuation and sensing of motion on the nanometer scale and recent nanopositioner designs have been utilizing microelectromechanical systems (MEMS). This brief demonstrates a simple method to implement vibration control on a MEMS nanopositioner. The actuation and sensing of the system are performed with a MEMS electrostatic drive. The electrostatic drive is arranged to be self-sensing, that is, the drive's voltage is used to actuate the system and the drive's current is used to observe the system. With this arrangement, the current is proportional to velocity at the resonance frequency and velocity feedback is used to damp the nanopositioner. To filter the current signal and recover a displacement signal, a charge measurement may be preferred to a current measurement. The self-sensing arrangement was modified to be a charge sensor and resonant control was applied to damp the nanopositioner. With this arrangement, the gain at the resonance frequency was attenuated by $18.45 \mathrm{~dB}$.
\end{abstract}

Index Terms-Electrostatic drive, microelectromechanical systems (MEMS), nanopositioning, self-sensing, vibration control.

\section{INTRODUCTION}

$\mathbf{N}$ ANOPOSITIONING is the actuation and control of motion in the order of nanometers and is a core aspect of nanotechnology [1]. Applications of nanopositioning include microscopy, data storage, and microfabrication [2], [3]. Recent nanopositioner designs have been using microelectromechanical systems (MEMS) technology [4]-[6]. MEMS devices are microscaled mechatronic systems produced using microfabrication techniques [7]. MEMS devices derive benefits from miniaturization, batch Production, and precision fabrication associated with the technology.

Transducers that exploit electrostatic, piezoelectric, thermal, magnetic [7], shape memory alloy [8], and phase change [9] phenomena have been shown suitable for incorporation into MEMS devices. Arguably, the most common transducers are electrostatic. An example is shown in Fig. 1. Their popularity stems from their compatibility with any conductive material, ease of fabrication and low power consumption. They consist of two electrodes in close proximity. When a potential difference is established between the two electrodes, an attractive force develops. The current that is drawn through the electrostatic drive is composed of a motional current and a feedthrough current [10]. The motional current is a function of the drive's velocity while the feedthrough current is associated with the capacitive nature of the electrostatic drive.

Manuscript received March 3, 2014; revised August 6, 2014; accepted October 8, 2014. Date of publication October 28, 2014; date of current version April 14, 2015. Manuscript received in final form October 9, 2014. Recommended by Associate Editor C. Natale.

The authors are with the School of Electrical Engineering and Compute Science, University of Newcastle, Callaghan, NSW 2308, Australia (e-mail: steven.i.moore@uon.edu.au; reza.moheimani@newcastle.edu.au).

Color versions of one or more of the figures in this paper are available online at http://ieeexplore.ieee.org.

Digital Object Identifier 10.1109/TCST.2014.2363137

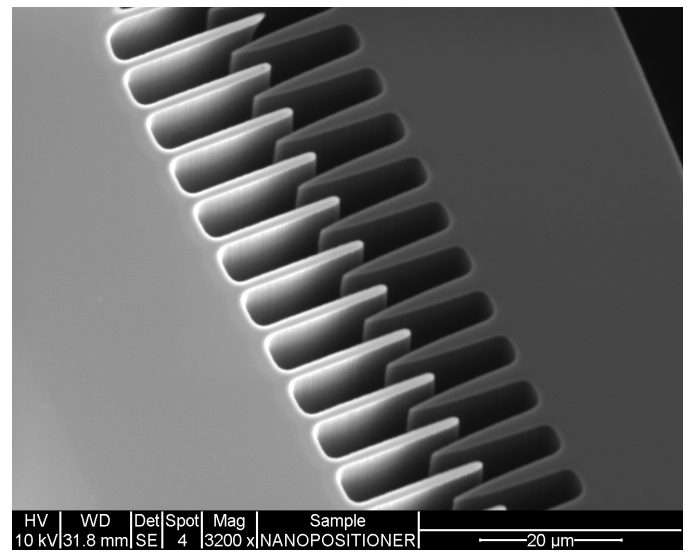

Fig. 1. Image of an electrostatic comb drive in a MEMS device. The fingerlike structure of the comb drive is used to give the transducer a linear characteristic with respect to displacement.

Sensing and control of the device's velocity can occur when the motional current dominates the feedthrough current. Current sensing for microelectronic systems is typically performed using a series resistance [11], [12], Wheatstone bridge [12], or transimpedance amplifier [10], [13]. Alternatively, integrating the current to produce a charge measurement has been performed to recover displacement signals and filter the current measurement [13], [14]. Several implementations of current sensing in piezoelectric transducers also cancel the feedthrough current, so the output is a function of velocity over all frequencies [11], [15].

Motivation for this brief comes from the extensive literature on macro-sized piezoelectric self-sensing transducers to create smart structures to damp vibrations [11], [13], [16]. In these systems, the voltage across the piezoelectric drive performs actuation, and the current or charge though it is used to observe the motion. With similarities existing between piezoelectric and electrostatic drives [10], self-sensing can also be applied to the latter. In MEMS, self-sensing would allow for the precious die space to be dedicated to one transducer, where previously two separate transducers would have been required. This effectively increases the transduction strength and simplifies the design of the MEMS device. Note there exists types of actuators that are suitable for self-sensing arrangements, for example, phase change actuators [17].

Mechanical structures typically consist of an infinite combination of lightly damped second-order modes. Being lightly damped, disturbance and impulses easily excite the modes of the mechanical structure. Vibration control is necessary in many applications for precision control and to protect the integrity of the mechanical structures [18], [19]. An array of control techniques have been applied to vibration control problems [19]. 
With the application of a self-sensing transducer, the systems attain a property called collocation [19], [20]. With displacement measurement, the system is negative imaginary [20], and with velocity measurement, the system is positive real [21]. There is a set of controllers commonly used with collocated systems [13], [18], [19], [22]. The popularity of these controllers stems mainly from their robust behavior when applied to collocated systems and also from their ease of design and effective performance [22].

Vibration control has been implemented in a range of devices for various purposes. Fairbairn and Moheimani [23] employs vibration control on atomic force microscopy (AFM) cantilevers. The quality factor of the cantilever is used to trade off between speed and resolution of an AFM scan. Sebastian et al. [24] employs vibration control in a MEMS data storage device. In addition to damping the structure, the resonant controller makes the system less sensitive to sensor noise. Gardonio et al. [25] applies vibration control in a MEMS velocity sensor to remove the resonant characteristic of its proof mass from its response. Liang et al. [26] uses control to tune the response of a MEMS accelerometer. By tuning a PID controller, the overshoot and settling time of the sensor were reduced.

This brief outlines the design of a simple current and charge self-sensing electrostatic drive and outlines its potential by performing feedback control on the nanopositioner as published in [5]. Two aspects of the electrostatic drive have provided motivation for this new design. First, with the $\mathrm{x}$ and $y$ drives sharing a common ground, current sensing must be performed on the high side. Second is the small magnitude of the currents drawn by electrostatic drives, typically less than tens of microamps.

The structure of this brief is as follows. Section II will introduce the nanopositioner and its transduction mechanisms and present modeling of the system. Section III outlines the design and performance of a current and charge sensor used to observe the motion of the nanopositioner. Section IV outlines a set of measurements that are used to identify the model's parameters and presents the input-output systems upon which control is to be applied. Section V considers the sensitivity, range, and spectral density of the current and charge sensor to detect the motion of the nanopositioner. Section VI provides an overview of the control topologies, controller structure, and the analysis to show how vibration control is implemented and what its effect is. With the fabricated system, Section VII shows the frequency responses from input voltage to displacement that validate the modeling of the nanopositioner and show the damping effect of the control.

\section{System Modeling}

The nanopositioner used for experimentation in this brief is shown in Fig. 2. The device is etched in a $25 \mu \mathrm{m}$ layer of doped single crystal silicon and rests on silicon substrate that is coated in silicon oxide. A trench is etched through the substrate to release the device. The fabrication is performed using the services of MEMSCAP.

Fig. 3 shows the model of one degree of freedom of the nanopositioner. The system is composed of a

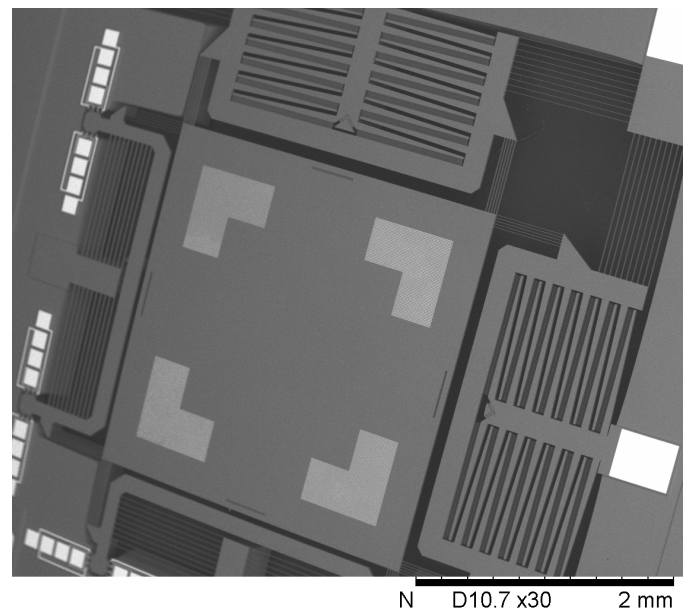

Fig. 2. Image of the nanopositioner upon which the experiments outlined in this brief are applied to. The electrostatic comb drives, upon which the self-sensing method is applied, can be seen to the right and top of the image.

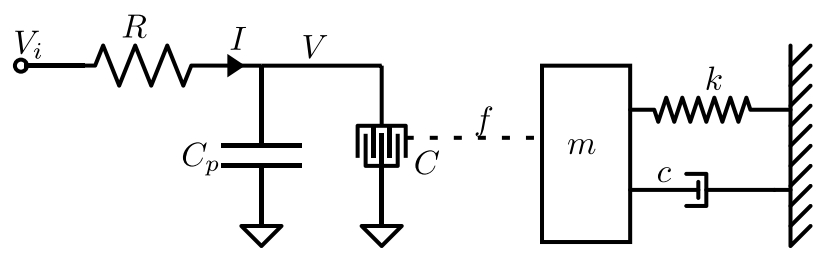

Fig. 3. Diagram of the electrical and mechanical systems that are coupled by an electrostatic drive. The electrostatic drive produces a force $f$ to actuate the nanopositioner's stage $m$.

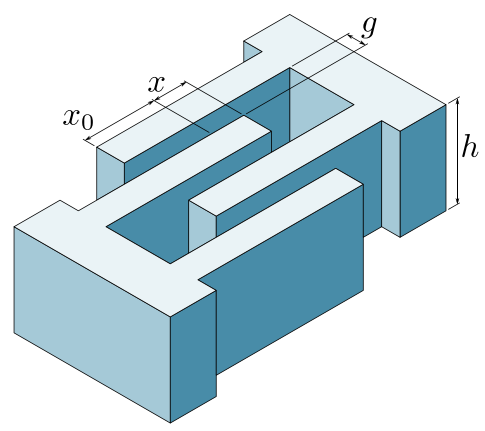

Fig. 4. Topology of a comb drive. The two electrodes consist of a set of intertwined fingers. They are typically constrained such that the electrode gap $g$ is constant and the electrode overlap $x$ changes. $x_{0}$ is defined as the zero voltage displacement and $h$ is the electrode height. Along with the number of overlapping electrode faces, these parameters are used to determine the transduction efficiency of the comb drive.

mechanical spring-mass-damper system and an electrical capacitive system [7]. The dynamics of these two systems are modeled by (1) and (2) as follows:

$$
\begin{aligned}
m \ddot{x}+c \dot{x}+k x & =f \\
i & =\frac{d}{d t}(C V) .
\end{aligned}
$$

Fig. 4 shows a portion of the comb drive used to actuate the system. One electrode is fixed while the other is constrained to move such that the electrode gap $g$ is constant while the electrode overlap $x$ changes. The capacitance of the comb drive is 


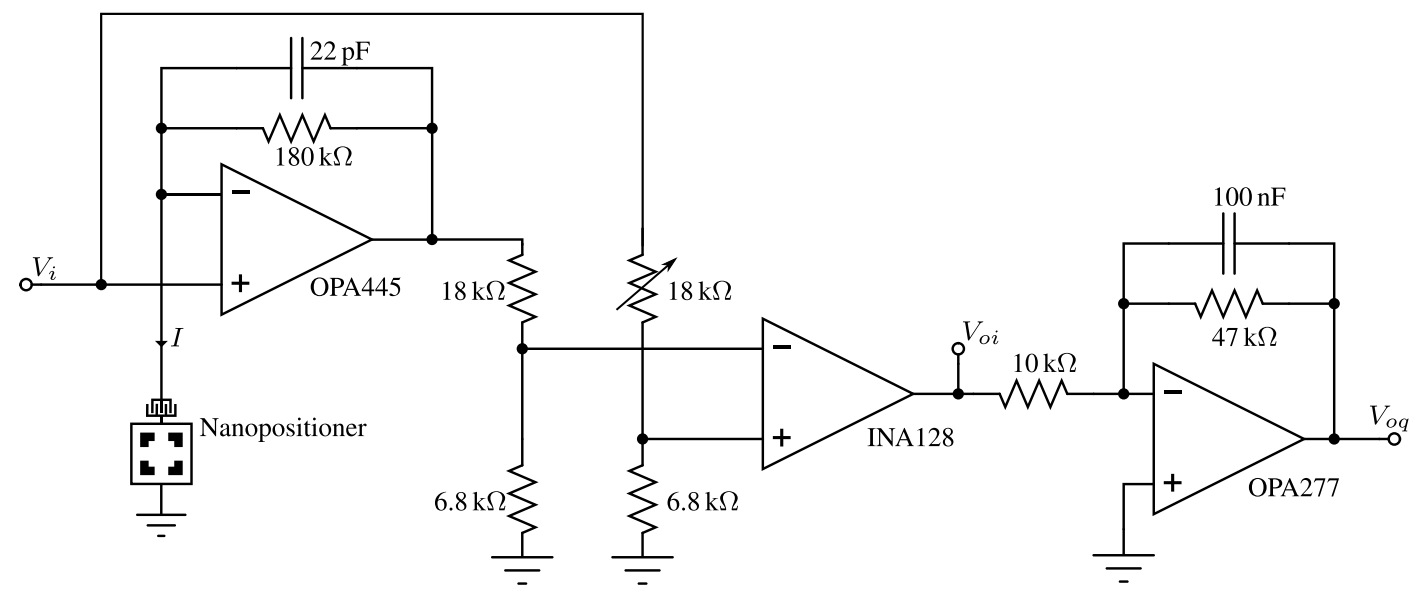

Fig. 5. Schematic of the current and charge sensor. A transimpedance amplifier is used to measure the current flowing into the capacitive drive. As it can operate at voltages above typical microelectronic levels, resistor dividers are used to shift the voltage level down. The current signal is read out using an instrumentation amplifier with a gain of 501. An integrator stage is added at the end to eliminate the differentiation characteristic in the current sensor. The output of the integrator is a charge measurement. All amplifiers are from Texas Instruments.

given in (3) below. For the comb drive of the nanopositioner, the number of overlapping electrode faces $n=3122$, the electrode height $h=25 \mu \mathrm{m}$, the dielectric permittivity $\varepsilon$ is the permittivity of free space, and the electrode gap $g=2 \mu \mathrm{m}$. The zero volt displacement $x_{0}=5 \mu \mathrm{m}$. The capacitance due to the zero volt displacement $x_{0}$ is incorporated into the parasitic capacitance $C_{p}$, because these two cannot be separately observed. The parameter $\alpha$ is defined to combine the constant terms in the coefficient of $x$

$$
C=\frac{n \varepsilon h}{g} x+C_{p}=\alpha x+C_{p} .
$$

The force is defined as the rate of change of capacitive energy with respect to displacement

$$
f=\frac{1}{2} V^{2} \frac{\partial C}{\partial x}=\frac{\alpha}{2} V^{2} .
$$

To make the system causal, the series resistance is taken into account. The current is expressed in terms of the drive voltage and input voltage

$$
i=\frac{V_{i}-V}{R} .
$$

Equations (1)-(5) are combined to form the dynamic model as shown in (6)-(8). The states of the system are the drive velocity $x_{1}=\dot{x}$, displacement $x_{2}=x$, and voltage $x_{3}=V$. The input to the system is the voltage $V_{i}$

$$
\begin{aligned}
& \dot{x_{1}}=\frac{1}{m}\left[-c x_{1}-k x_{2}+\frac{1}{2} \alpha x_{3}^{2}\right] \\
& \dot{x_{2}}=x_{1} \\
& \dot{x_{3}}=\frac{V_{i}-x_{3}-\alpha R x_{1} x_{3}}{\alpha R x_{2}+R C_{p}} .
\end{aligned}
$$

The system is biased by setting the input voltage to a value of $V_{0}$. This biases the states at $x_{1}=0, x_{2}=(\alpha / 2 k) V_{0}^{2}$, and $x_{3}=V_{0}$. The system is linearized around this bias point. The state space model is as follows. The input $u=V_{i}$ and the output of the system is the current $y=I$

$$
\begin{aligned}
& \dot{x}=A x+B u \\
& y=C x+D u
\end{aligned}
$$

where

$$
\begin{aligned}
A & =\left[\begin{array}{ccc}
-\frac{c}{m} & -\frac{k}{m} & \frac{\alpha}{m} V_{0} \\
1 & 0 & 0 \\
-\frac{2 k \alpha V_{0}}{\alpha^{2} V_{0}^{2}+2 k C_{p}} & 0 & -\frac{2 k}{\alpha^{2} R V_{0}^{2}+2 k R C_{p}}
\end{array}\right] \\
B & =\left[\begin{array}{c}
0 \\
0 \\
\frac{2 k}{\alpha^{2} R V_{0}^{2}+2 k R C_{p}} u
\end{array}\right] \\
C & =\left[\begin{array}{lll}
0 & 0 & -\frac{1}{R}
\end{array}\right] \\
D & =\frac{1}{R} .
\end{aligned}
$$

\section{Self-Sensing Design}

The current drawn by the electrostatic drive is measured for use in feedback control. The schematic used to measure current is shown in Fig. 5. The scheme uses a high side transimpedance amplifier. The transimpedance amplifier is used on the high side because there are multiple drives connected to a common ground on the MEMS device. The disadvantage of the high side sensing is that the output is the sum of the actuation signal as well as the current signal. Resister dividers shift the voltage levels down and they are read out by an instrumentation amplifier.

An issue with this design is the requirement that resistor dividers be perfectly balanced. This prevents the actuation voltage from feeding through the instrumentation amplifier. This zeroing is performed with a potentiometer on the high side of one of the resistor dividers. Because no dc current flows through electrostatic drives, any dc voltage that appears at the circuit output is due to mismatch between the voltage dividers. Therefore, zeroing any dc offset that appears at the output of the sensor will zero the circuit.

Modeling the current sensor, the low frequency gain from the current $I$ to the current sensor output $V_{\text {oi }}$ is $-24.72 \times$ $10^{6} \mathrm{VA}^{-1}$ and the bandwidth is $40.2 \mathrm{kHz}$.

Because of the capacitive nature of the electrostatic drive, this scheme functions as a differentiator from the input 


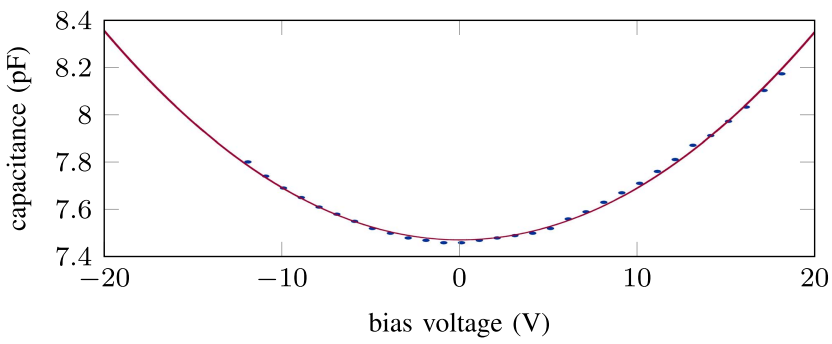

Fig. 6. Capacitance of the drive for an applied bias voltage. The measurements and a fitted curve are shown. The equation of the capacitance in $\mu \mathrm{m}$ is $0.002199 \mathrm{~V}^{2}+7.472$

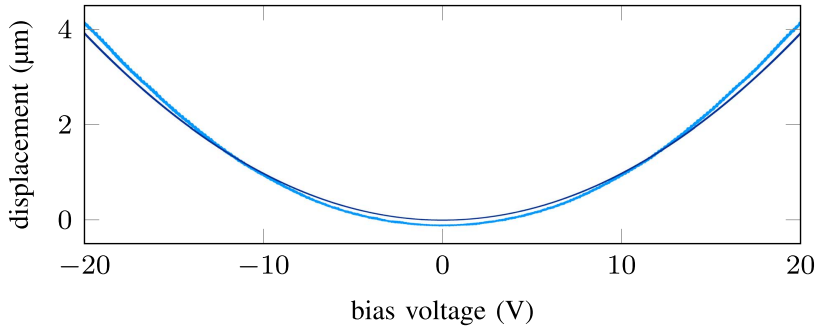

Fig. 7. Displacement of the drive for an applied bias voltage. The measurements and a fitted curve are shown. The equation for the displacement is in $\mu \mathrm{m}$ is $0.009798 \mathrm{~V}^{2}$.

voltage $V_{i}$ to the current sensor output $V_{\mathrm{oi}}$. To remove the differentiator characteristic, an integrator stage is added to the system. The output $V_{\text {oq }}$ is in effect a charge measurement. Equation (15) contains the transfer function of the integrator

$$
F=-\frac{4.7}{0.0047 \mathrm{~s}+1} \text {. }
$$

The mapping from charge $Q$ to voltage output $V_{\mathrm{oq}}$ in the passband of the charge sensor has a gain of $24.72 \mathrm{GVC}^{-1}$. The passband of the charge measurement is from $33.9 \mathrm{~Hz}$ to $40.2 \mathrm{kHz}$.

The nature of the first op amp is important. The op amp must be able to supply the voltages required by the nanopositioner. Second, owing to the small currents that flow into the system and the high gain of the transimpedance amplifier, an op amp that draws small bias currents is of benefit. The OPA445 op amp from Texas Instruments is one such device suitable for this application.

\section{SYSTEM IDENTIFICATION}

The capacitance and the resistance of the system were measured using an LCR meter. The resistance was $R=353 \Omega$. The capacitance as a function of bias voltage is shown in Fig. 6.

The other static characterization of the device is the displacement of the stage as a function of an applied voltage which is shown in Fig. 7. Using the fitted curves from Figs. 6 and 7, the mapping between capacitance and displacement is found as $\alpha=0.2244 \times 10^{-6} \mathrm{Fm}^{-1}$ and the stiffness is found as $k=11.45 \mathrm{Nm}^{-1}$.

The frequency response from voltage to the current sensor output is used to characterize the rest of the system and is shown in Fig. 8. The complex poles and zeros are easily
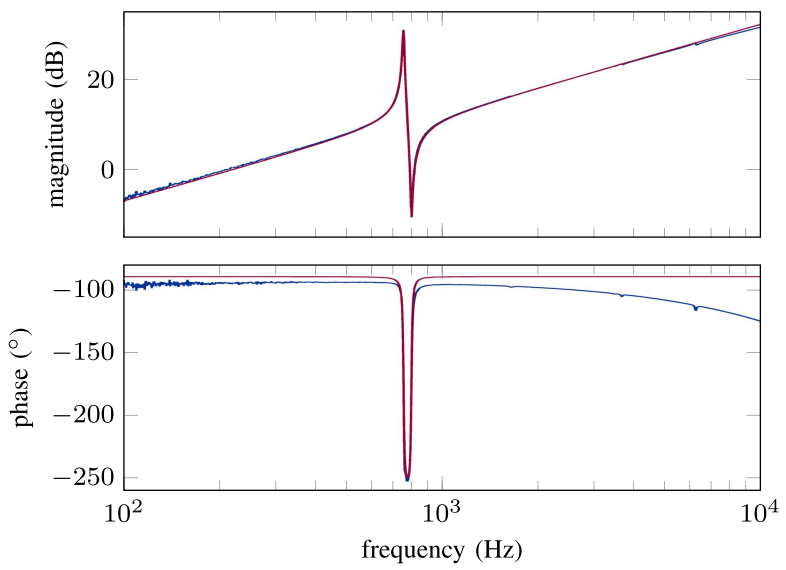

Fig. 8. Frequency response from the input voltage $V_{i}$ to the current sensor output voltage $V_{\mathrm{oi}}$. The system is biased at $25 \mathrm{~V}$. Shown is the measured frequency response (blue) compared with the modeled frequency response (red). The current sensor operates like a voltage differentiator at higher frequencies amplifying noise.

identified from a fitted model. The complex poles have a natural frequency of $757 \mathrm{~Hz}$ and a quality factor of 93. The complex zeros have a frequency of $803 \mathrm{~Hz}$. Using the value of the complex poles, the mass $m=506 \times 10^{-9} \mathrm{~kg}$ and the damping coefficient $c=25.92 \times 10^{-6} \mathrm{Nsm}^{-1}$ are determined. Using the value of the complex zeros, the parasitic capacitance is found to be $\mathrm{Cp}=21.0 \mathrm{pF}$. By equating the low frequency gain of the transfer function to the value measured at $100 \mathrm{~Hz}$, the sensor gain is determined as $-28.65 \mathrm{MVA}^{-1}$.

The parameters identified in this section are combined with the model derived in Section II. The transfer function from input voltage to the current sensor output is shown in (16). The frequency response of this transfer function is shown in Fig. 8

$$
G_{1}(s)=\frac{-81166 s\left(s^{2}+51.24 s+2.542 \times 10^{7}\right)}{\left(s+1.266 \times 10^{8}\right)\left(s^{2}+51.26 s+2.264 \times 10^{7}\right)} .
$$

The transfer function of the integrator, shown in (15), is appended to the transfer function $G_{1}$ in (16). This produces the mapping from this input voltage to the charge sensor output as shown in (17). The frequency response of this transfer function is shown in Fig. 9

$$
\begin{aligned}
& G_{2}(s) \\
& =\frac{8.117 \times 10^{7} s\left(s^{2}+51.24 s+2.542 \times 10^{7}\right)}{\left(s+1.266 \times 10^{8}\right)(s+212.8)\left(s^{2}+51.26 s+2.264 \times 10^{7}\right)} .
\end{aligned}
$$

Not all dynamics are modeled and identified in this brief. This can be seen in the discrepancies between the measured and modeled frequency responses as shown in Figs. 8 and 9. Unmodeled dynamics include the bandwidth of the sensor and the feedthrough of the input voltage. Importantly, the modeling of the resonant dynamics corresponds closely to the measured dynamics. This allows for the design of feedback controllers to implement vibration control. 

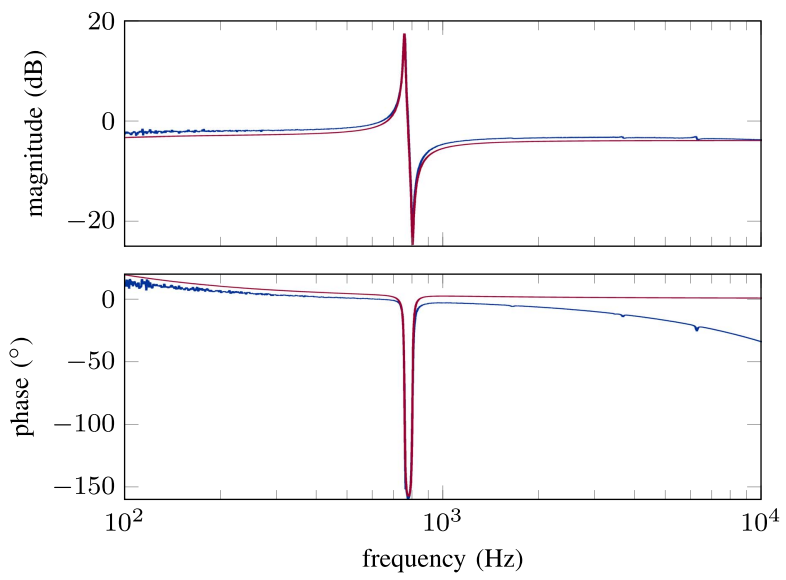

Fig. 9. Frequency response from the input voltage $V_{i}$ to the charge sensor output voltage $V_{\mathrm{oq}}$. Shown is the measured frequency response (blue) compared with the modeled frequency response (red). While the gain at resonance is lower than the case with the current sensor, it no longer amplifies the voltage noise at higher frequencies.

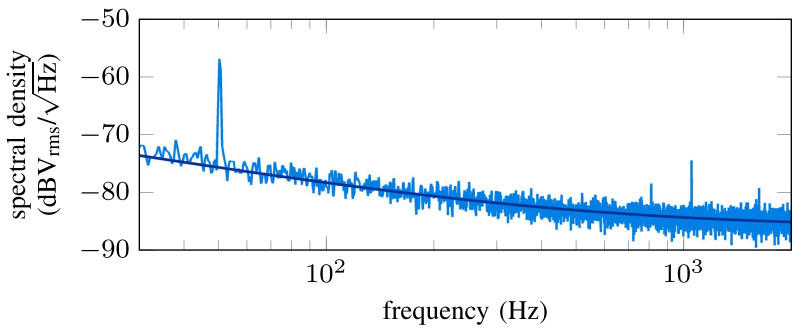

Fig. 10. Shown is the spectral density at the output of the current sensor. The noise model (the darker trace) contains flicker noise and white noise The flicker noise has a corner frequency of $492 \mathrm{~Hz}$ and the noise floor is at $-85.99 \mathrm{~dB}$.

\section{Velocity And Displacement SEnsing}

This section will consider the performance of the sensing method to detect motion of the nanopositioner. The metrics of sensitivity, spectral density, and range are reported.

Under the conditions that the motional current dominates the feedthrough current and the changes in voltage are small, the current is proportional to the velocity of the drive, as shown in

$$
i=V_{0} \alpha \dot{x} .
$$

Considering the gain of the current sensor $A_{i}=$ 28.65 $\mathrm{MVA}^{-1}$, the gain from velocity to current sensor output is $A_{v}=160.8 \mathrm{Vs} \mathrm{m}^{-1}$.

With the nanopositioner removed and the transimpedance amplifier biased at $25 \mathrm{~V}$, the spectral density was measured at the output of the current sensor, as shown in Fig. 10. Both flicker noise and white noise are considered present and the noise model in (19) is fitted to the measured spectral density. The fitted model has a noise floor of $A=-85.99 \mathrm{~dB}$ and noise corner frequency of $f_{\mathrm{nc}}=492 \mathrm{~Hz}$. The spectral density noise floor of this spectrum is $50.18 \mu \mathrm{V}_{\mathrm{rms}} / \sqrt{\mathrm{Hz}}$

$$
S(f)=A \sqrt{\frac{f_{\mathrm{nc}}}{f}+1} .
$$

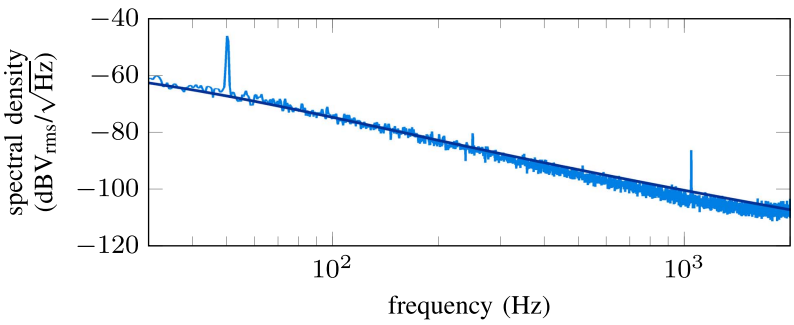

Fig. 11. Shown is the spectral density at the output of the charge sensor. The fitted noise model is the product of the current sensor noise model and the response of the lossy integrator appended to make the charge measurement.

Taking the velocity sensor gain of $A_{v}=160.8 \mathrm{~V} \mathrm{sm}^{-1}$, the velocity noise floor is at $-130.1 \mathrm{~dB}$. That is a spectral density of $312 \mathrm{~nm} / \mathrm{s} \sqrt{\mathrm{Hz}}$.

Integrating the expression of the motional current in (18) provides an expression for the motional charge, as shown in (20). It is proportional to the displacement of the drive

$$
q=V_{0} \alpha x .
$$

Considering the gain of the charge sensor $A_{q}=28.65 \mathrm{GVC}^{-1}$, the sensitivity of the displacement measurement is $A_{d}=$ $160.8 \mathrm{kV} \mathrm{m}^{-1}$.

Fig. 11 shows the spectral density at the output of the charge sensor. The charge sensor is the current sensor plus the addition of a lossy integrator whose model $F(s)$ is given in (15). Therefore, the spectral density can be accounted for as the product of $|F(j 2 \pi f)|$ and the spectral density of the current sensor $S(f)$ in (19). The noise floor of this charge sensor was observed at $-107 \mathrm{~dB}$. The spectral density noise floor of this spectrum is $4.47 \mu \mathrm{V}_{\mathrm{rms}} / \sqrt{\mathrm{Hz}}$.

Considering the displacement sensitivity of $A_{d}=$ $160.8 \mathrm{kV} \mathrm{m}^{-1}$, the displacement noise floor is at $-211.1 \mathrm{~dB}$ or $27.8 \mathrm{pm} / \sqrt{\mathrm{Hz}}$.

Saturation of the instrumentation amplifier with a $\pm 15 \mathrm{~V}$ power supply provides the limit of both sensors. Considering the sensitivity of the current sensor $A_{i}=28.65 \mathrm{MV} \mathrm{A}^{-1}$, the current range of the sensor is $\pm 524 \mathrm{nA}$. Therefore, the range of the velocity sensor is $\pm 93.4 \mathrm{~mm} \mathrm{~s}^{-1}$.

Another issue is the substantial noise at low frequencies. A number of causes can be attributed to this. First, the integrator in the final stage amplifies low frequencies. Changing the transimpedance amplifier to a charge amplifier removes the necessity of this integrator stage. Second, the transimpedance amplifier feeds through the actuation signal to the instrumentation amplifier. The noise on this signal would also feed through. A design with a better common mode rejection would be of benefit. Third, the sensors are dc coupled. With no current or charge flowing at zero frequency, ac coupling should be considered in the system to reduce noise.

\section{Active Damping of the NANopositioner}

Active damping will be implemented with the control topology as shown in Fig. 12. Active damping with a proportional controller requires a phase shift of $0^{\circ}$ at resonance in the plant. This phase shift is already achieved when using the current 


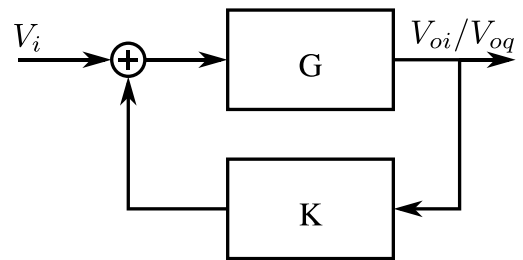

Fig. 12. Control topology implementing vibration control of the nanopositioner. For different sensor outputs, the controller $K$ will differ.

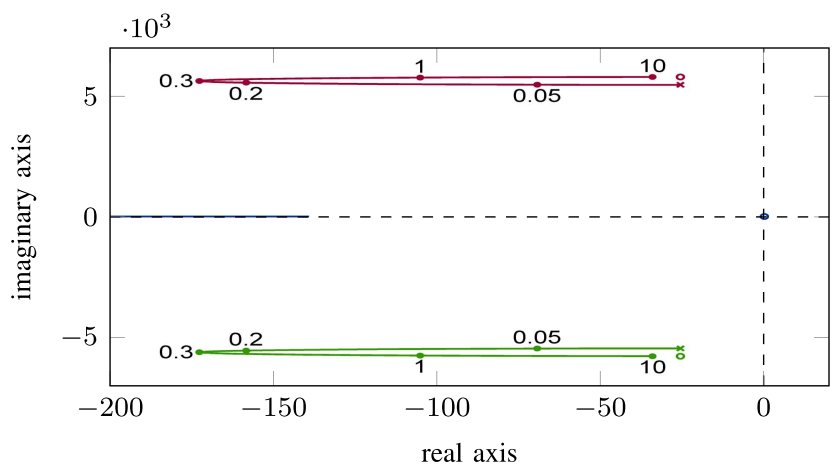

Fig. 13. Plots show the loci of the poles of the closed loop current sensor system as the controller gain is increased. With no gain, the quality factor of the complex poles is 93 . Using the current sensor and a proportional controller, a gain of 0.31 reduces quality factor of the complex poles to 14.3.

sensor system as shown in Fig. 8. When using the charge sensor system as shown in Fig. 9, a phase shift of $\pm 90^{\circ}$ needs to be introduced.

The gain of the controller is increased to damp the system. Increasing the gain moves the closed loop poles toward the zeros of the system. Above a certain gain, the resonant motion of the device will become less observable. Thus, the active damping will become less effective. A different control architecture would be needed for increased damping.

For the current sensor system $G_{1}$ in (16), a proportional controller is used to perform vibration control. The loci of the closed loop poles are shown in Fig. 13. The quality factor of the open loop complex poles is 93. The loci are plotted for gains from 0 to 10 in positive feedback. Maximum damping with this controller topology occurs when the gain is 0.31 . The quality factor at this point is 14.3. After this gain, the closed loop poles move toward the zeros and the damping effect decreases.

When using the charge sensor system $G_{2}$ in (17), a resonant controller $K$ is used to achieve a $90^{\circ}$ phase shift at the resonance frequency. The controller's natural frequency is tuned to the system natural frequency to ensure the phase shift occurs at the correct frequency. Equation (21) is the transfer function of the resonant controller

$$
K(s)=\frac{s^{2}}{s^{2}+4750 s+2.256 \times 10^{7}} .
$$

The loci of the closed loop poles of the loop gain $G_{2} K$ are shown in Fig. 14. The plot shows the locus for gains $0-10$ in negative feedback. Maximum damping occurs when the gain of the controller is 1.28. At this point, the quality factor of the closed loop poles is 14.4.

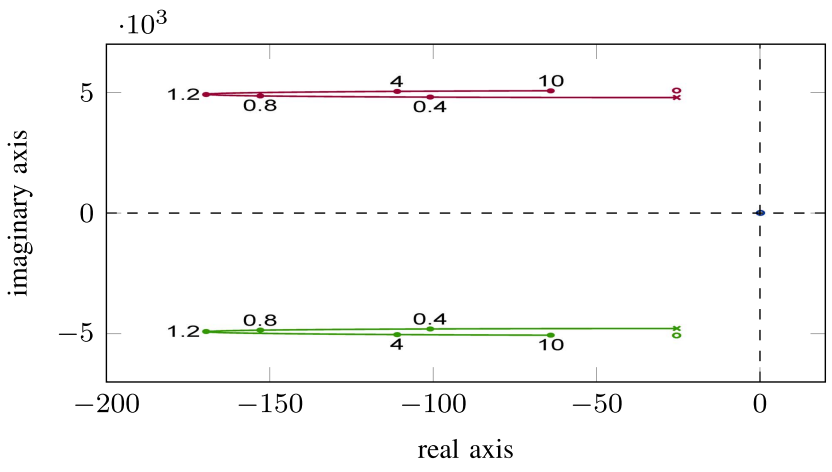

Fig. 14. Plots show the loci of the poles of the closed loop charge sensor system as the controller gain is increased. With no gain, the quality factor of the complex poles is 93 . With the charge sensor and resonant controller, a gain of 1.28 reduces the quality factor of the complex poles to 14.4.

\section{RESULTS}

A circuit board was fabricated to implement the nanopositioner with the self-sensing drive. As noted in Section VI, vibration control of the current sensor system can be implemented with proportional feedback owing to the $180^{\circ}$ phase shift at resonance. Vibration control of the charge sensor system can be implemented with a resonant controller because of the $-90^{\circ}$ phase shift at resonance. The controllers are implemented using a dSPACE controller board. The displacement measurements in this section are made with a laser Doppler vibrometer.

Using the current sensor, the system was incorporated into a positive feedback control topology. A controller with a band pass characteristic was used to actively damp the resonant motion of the system. The bandpass characteristic is used to prevent the controller acting at frequencies other than the resonance frequency. This was deemed necessary due to the current sensor amplifying noise at higher frequencies, the noise associated with the controller prototyping system and unmodeled dynamics at higher frequencies. A second-order bandpass filter with a quality factor of 1 was implemented. The transfer function of the controller $K_{1}$ is stated in (22). The gain at the resonance frequency $k$, determines the strength of the damping

$$
K_{1}(s)=k \frac{4750 s}{s^{2}+4750 s+2.256 \times 10^{7}} .
$$

Using the charge sensor, the system was incorporated into a negative feedback control structure. A resonant controller was implemented to damp the resonant motion. The transfer function of the resonant controller is shown in

$$
K_{2}(s)=k \frac{s^{2}}{s^{2}+4750 s+2.256 \times 10^{7}} .
$$

Using the current sensor with velocity feedback, Fig. 15 shows the frequency response from the input voltage to the displacement. Progressive damping in the frequency response is obtained by increasing the gain of the controller. Gains of $0.05,0.15,0.25$, and 0.3 were used. Damping of $19.27 \mathrm{~dB}$ was achieved.

Using the charge sensor with resonant control, Fig. 16 shows the frequency response from input voltage to displacement. 

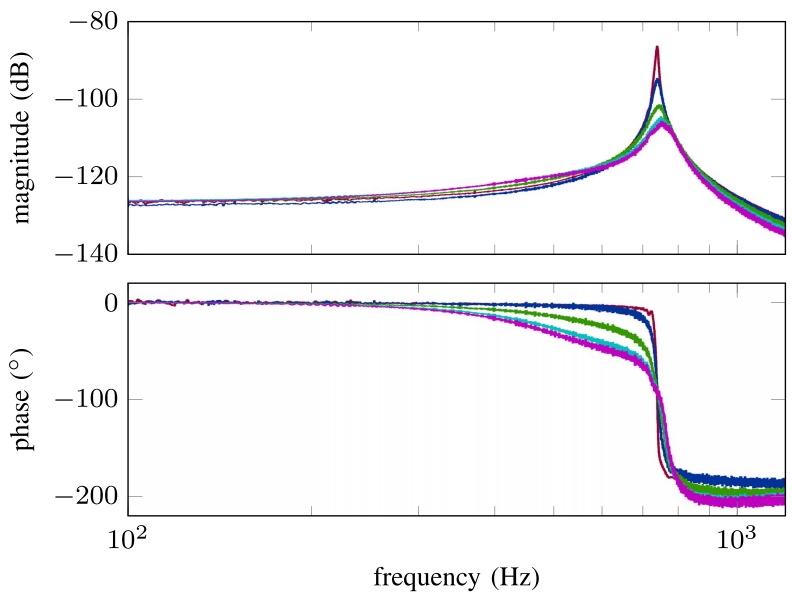

Fig. 15. Measured frequency response from the input voltage to the displacement with velocity feedback using the current measurement. The most undamped response is the open loop response. For progressively more damping, the controller gain $k$ used was $0.05,0.15,0.25$, and 0.3 . An attenuation of $19.27 \mathrm{~dB}$ was achieved with $k=0.3$.
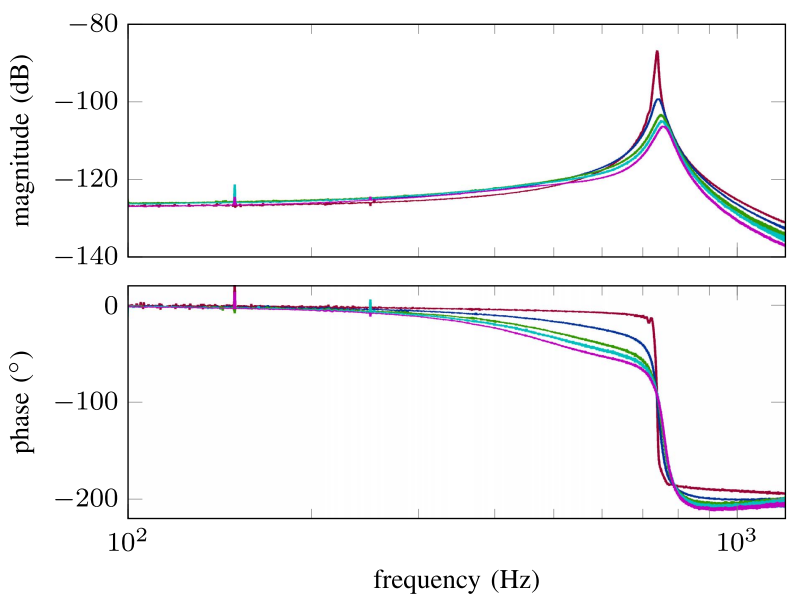

Fig. 16. Measured frequency response from the input voltage to the displacement with resonant control using the charge measurement. The most undamped response is the open loop response. For progressively more damping, the controller gain $k$ used was $0.4,0.8,1$, and 1.2. An attenuation of $18.45 \mathrm{~dB}$ was achieved with $k=1.2$.

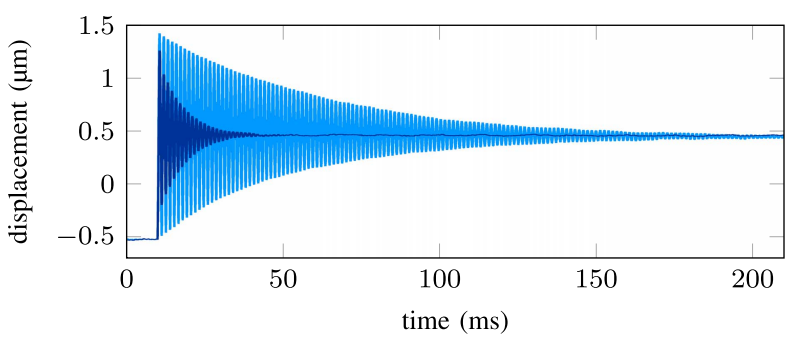

Fig. 17. Step response of the open loop and closed loop systems. These responses demonstrate the effect of the vibration control. The closed loop system is implemented with the charge sensor and a resonant controller with a gain of 1.2. The system is stepped by $1 \mathrm{~V}$.

Progressive damping was achieved with gains of $0.4,0.8$, 1 and 1.2. Damping of $18.45 \mathrm{~dB}$ was achieved.

To show the effectiveness of the vibration control, step responses of the open loop and closed loop systems are compared as shown in Fig. 17. The closed loop system uses a charge sensor and a resonant controller with a gain of 1.2. It shows the closed loop system settles significantly faster than the open loop system.

\section{CONCLUSION}

This brief has outlined the modeling, identification, and design of a self-sensing method applied to a MEMS electrostatic drive. Vibration control was applied to the system to demonstrate a possible application of the self-sensing method. The self-sensing method utilizes a current sensor and charge sensor. Using the charge sensor output and a resonant controller, the resonant mode of a MEMS nanopositioner was attenuated by $18.45 \mathrm{~dB}$.

The design presented can be easily used in devices requiring vibration control. However, there are few aspects of the design that warrant consideration or improvement.

Because of the differentiator characteristic in the current sensor, it is recommend to use the charge sensor to observe the system. Simplifications in the implementation are possible with the elimination of the current sensor.

The presented design feeds through some of the actuation signal to the sensor output and no current flows at zero frequency. A design with improved common mode rejection and ac coupling would improve the offset, drift, and noise.

If it is desired to control frequencies off the resonance frequency or if resonant motion in the system is small, feedthrough cancellation would need to be implemented.

In this brief, only one drive of the nanopositioner was actuated at once. There exists coupling in the electrical and mechanical systems. The extent of the coupling may need to be considered in controller design.

The validity of the linearization would need to be considered in any design. With lower bias voltages, the motional current becomes smaller. The implications are that for lower bias voltages, a high gain controller would be needed and the motional current becomes less observable compared with the feedthrough current. This places a lower limit on operating voltages in which the presented sensing and control scheme would remain effective. Solutions to linearize the system would be considered as an improvement. A possible solution is to use electrostatic drives in a push-pull arrangement [6] whereby the combination of the current from both drives would be used for sensing.

This method provides a simple implementation to perform self-sensing actuation on MEMS electrostatic drives. The scheme only requires one terminal of the electrostatic drive, allowing it to be used in systems where multiple drives are connected to a common ground.

\section{REFERENCES}

[1] S. Devasia, E. Eleftheriou, and S. O. R. Moheimani, "A survey of control issues in nanopositioning," IEEE Trans. Control Syst. Technol., vol. 15, no. 5, pp. 802-823, Sep. 2007.

[2] N. B. Hubbard, M. L. Culpepper, and L. L. Howell, "Actuators for micropositioners and nanopositioners," Appl. Mech. Rev., vol. 59, no. 6, pp. 324-334, Nov. 2006.

[3] B. Sahu, K. K. Leang, and C. R. Taylor, "Emerging challenges of microactuators for nanoscale positioning, assembly, and manipulation," J. Manuf. Sci. Eng., vol. 132, no. 3, p. 030917, 2010. 
[4] L. Sun, J. Wang, W. Rong, X. Li, and H. Bao, "A silicon integrated micro nano-positioning $X Y$-stage for nano-manipulation," J. Micromech. Microeng., vol. 18, no. 12, p. 125004, 2008.

[5] A. G. Fowler, A. N. Laskovski, A. C. Hammond, and S. O. R. Moheimani, "A 2-DOF electrostatically actuated MEMS nanopositioner for on-chip AFM," J. Microelectromech. Syst., vol. 21, no. 4, pp. 771-773, Aug. 2012.

[6] M. Maroufi and S. O. R. Moheimani, "Design, fabrication and characterization of a high-bandwidth 2DOF MEMS nanopositioner," in Proc. IEEE/ASME Int. Conf. Adv. Intell. Mechatron., Wollongong, N.S.W., Australia, Jul. 2013, pp. 335-340.

[7] C. Liu, Foundations of MEMS. Englewood Cliffs, NJ, USA: Prentice-Hall, 2011

[8] D. J. S. Ruth, S. S. Nakshatharan, and K. Dhanalakshmi, "Differential resistance feedback control of a self-sensing shape memory alloy actuated system," ISA Trans., vol. 53, no. 2, pp. 289-297, 2014.

[9] S. Ogden, L. Klintberg, G. Thornell, K. Hjort, and R. Bodén, "Review on miniaturized paraffin phase change actuators, valves, and pumps," Microfluidics Nanofluidics, vol. 17, no. 1, pp. 53-71, 2014.

[10] V. Kaajakari, Practical MEMS. Warsaw, Poland: Small Gear Publishing, 2009.

[11] J. J. Dosch, D. J. Inman, and E. Garcia, "A self-sensing piezoelectric actuator for collocated control," J. Intell. Mater. Syst. Struct., vol. 3, no. 1, pp. 166-185, 1992.

[12] K. Janschek, Mechatronic Systems Design: Methods, Models, Concepts. New York, NY, USA: Springer-Verlag, 2012.

[13] S. O. R. Moheimani, "A survey of recent innovations in vibration damping and control using shunted piezoelectric transducers," IEEE Trans. Control Syst. Technol., vol. 11, no. 4, pp. 482-494, Jul. 2003.

[14] E. Grasso, N. Totaro, H. Janocha, and D. Naso, "Piezoelectric self sensing actuators for high voltage excitation," Smart Mater. Struct., vol. 22, no. 6, p. 065018, 2013.

[15] J. E.-Y. Lee and A. A. Seshia, "Parasitic feedthrough cancellation techniques for enhanced electrical characterization of electrostatic microresonators," Sens. Actuators A, Phys., vol. 156, no. 1, pp. 36-42, 2009.
[16] S. Kuiper and G. Schitter, "Active damping of a piezoelectric tube scanner using self-sensing piezo actuation," Mechatronics, vol. 20, no. 6 , pp. $656-665,2010$.

[17] E. Merced, D. Torres, X. Tan, and N. Sepulveda, "An electrothermally actuated $\mathrm{VO}_{2}$-based MEMS using self-sensing feedback control," J. Microelectromech. Syst., vol. PP, no. 99, p. 1, May 2014.

[18] S. O. R. Moheimani and A. J. Fleming, Piezoelectric Transducers for Vibration Control and Damping. New York, NY, USA: Springer-Verlag, 2006.

[19] A. Preumont, Vibration Control of Active Structures. Amsterdam, The Netherlands: Springer-Verlag, 2011.

[20] I. R. Petersen and A. Lanzon, "Feedback control of negativeimaginary systems," IEEE Control Syst., vol. 30, no. 5, pp. 54-72, Oct. 2010.

[21] B. Brogliato, R. Lozano, B. Maschke, and O. Egeland, Dissipative Systems Analysis and Control: Theory and Applications. London, U.K.: Springer-Verlag, 2007.

[22] S. S. Aphale, A. J. Fleming, and S. O. R. Moheimani, "Integral resonant control of collocated smart structures," Smart Mater. Struct., vol. 16, no. 2, p. 439, 2007.

[23] M. Fairbairn and S. O. R. Moheimani, "Sensorless enhancement of an atomic force microscope micro-cantilever quality factor using piezoelectric shunt control," Rev. Sci. Instrum., vol. 84, no. 5, p. 053706 , May 2013.

[24] A. Sebastian, A. Pantazi, S. O. R. Moheimani, H. Pozidis, and E. Eleftheriou, "Achieving subnanometer precision in a MEMS-based storage device during self-servo write process," IEEE Trans. Nanotechnol., vol. 7, no. 5, pp. 586-595, Sep. 2008.

[25] P. Gardonio, M. Gavagni, and A. Bagolini, "Seismic velocity sensor with an internal sky-hook damping feedback loop," IEEE Sensors J., vol. 8, no. 11, pp. 1776-1784, Nov. 2008.

[26] Y. Liang, L. Xiaowei, C. Weiping, and Z. Zhiping, "High resolution interface circuit for closed-loop accelerometer," J. Semicond., vol. 32, no. 4 , p. 045005,2011 